\title{
Assessment of Subclinical Vascular Disease Associated with Ankylosing Spondylitis
}

\author{
NÓRA BODNÁR, GYÖRGY KEREKES, ILDIKÓ SERES, GYÖRGY PARAGH, JÁNOS KAPPELMAYER, \\ ZSUZSANNA GYURCSIK NÉMETHNÉ, GYULA SZEGEDI, YEHUDA SHOENFELD, SÁNDOR SIPKA, \\ PÁL SOLTÉSZ, ZOLTÁN SZEKANECZ, and SÁNDOR SZÁNTÓ
}

\begin{abstract}
Objective. Studies indicate that ankylosing spondylitis (AS), as well as rheumatoid arthritis, may be associated with accelerated atherosclerosis and vascular disease. We assessed endothelial dysfunction, carotid atherosclerosis, and aortic stiffness in AS in context with clinical and laboratory measurements.

Methods. Forty-three patients with AS and 40 matched healthy controls were studied. We assessed common carotid intima-media thickness (ccIMT), flow-mediated vasodilation (FMD), and pulse-wave velocity (PWV) in association with age, disease duration, smoking habits, body mass index, patient's assessment of pain and disease activity, Bath AS Disease Activity Index, Bath AS Functional Index (BASFI), metric measurements, erythrocyte sedimentation rate, C-reactive protein, and HLA-B27 status.

Results. We found impaired FMD $(6.85 \pm 2.98 \%$ vs $8.30 \pm 3.96 \% ; \mathrm{p}=0.005)$, increased ccIMT $(0.65$ \pm 0.15 vs $0.54 \pm 0.15 \mathrm{~mm} ; \mathrm{p}=0.01)$, and higher PWV $(8.64 \pm 2.44$ vs $8.00 \pm 1.46 \mathrm{~m} / \mathrm{s} ; \mathrm{p}=0.03)$ in patients with AS compared to controls, respectively. We also found that ccIMT negatively correlated with FMD $(r=-0.563 ; p=0.0001)$ and positively correlated with PWV $(r=0.374 ; p=0.018)$. Both ccIMT and PWV correlated with disease duration $(\mathrm{r}=0.559 ; \mathrm{p}=0.013$ and $\mathrm{r}=0.520 ; \mathrm{p}=$ 0.022 , respectively), BASFI $(r=0.691 ; p=0.003$ and $r=0.654 ; p=0.006)$, decreased lumbar spine mobility $(\mathrm{r}=-0.656 ; \mathrm{p}=0.006$ and $\mathrm{r}=-0.604 ; \mathrm{p}=0.013)$, chest expansion $(\mathrm{r}=-0.502 ; \mathrm{p}=0.047$ and $\mathrm{r}=-0.613 ; \mathrm{p}=0.012)$, and increased wall-occiput distance $(\mathrm{r}=0.509 ; \mathrm{p}=0.044$ and $\mathrm{r}=0.614$; $\mathrm{p}=0.011$ ).

Conclusion. In this well characterized AS population, impaired FMD and increased ccIMT and PWV indicate abnormal endothelial function and increased atherosclerosis and aortic stiffness, respectively. The value of noninvasive diagnostic tools needs to be further characterized. (First Release Jan 15 2011; J Rheumatol 2011;38:723-9; doi:10.3899/jrheum.100668)
\end{abstract}

Key Indexing Terms:

ANKYLOSING SPONDYLITIS

ENDOTHELIAL DYSFUNCTION

FLOW-MEDIATED VASODILATION

\section{CARDIOVASCULAR DISEASE ATHEROSCLEROSIS ARTERIAL STIFFNESS}

Accelerated atherosclerosis and increased cardiovascular morbidity and mortality have been associated with various inflammatory rheumatic diseases. Most data have become available in rheumatoid arthritis (RA) $)^{1,2,3,4,5}$. Much less information is available regarding ankylosing spondylitis $(\mathrm{AS})^{3,6,7,8,9,10,11,12,13,14,15,16,17,18,19,20,21}$. There is a clinically relevant increase in the prevalence of cardiovascular diseases and higher cardiovascular mortality in $\mathrm{AS}^{22,23}$. The
From the Department of Rheumatology, Institute of Medicine;

Cardiovascular Unit and Laboratory of Immunology, Third Department of Medicine, Institute of Medicine; Division of Metabolic Diseases, First Department of Medicine; Department of Clinical Biochemistry and Molecular Pathology, and the Department of Physiotherapy, University of Debrecen Medical and Health Science Center (MHSC); Research Center for Autoimmune Diseases, Hungarian Academy of Sciences, Debrecen, Hungary; and Department of Medicine B and Center for Autoimmune Diseases, Sheba Medical Center, Tel-Hashomer, Israel.

Supported by research grants ETT 315/09 from the Hungarian Medical Research Council, T 046517 from the National Foundation for Scientific Research (OTKA), a Bolyai Research Grant, and a research grant from the Hungarian Academy of Sciences.

N. Bodnár, MD, Department of Rheumatology, Institute of Medicine; G. Kerekes, MD, Cardiovascular Unit; I. Seres, MD, PhD, Division of Metabolic Diseases, First Department of Medicine; G. Paragh, MD, PhD, Division of Metabolic Diseases, First Department of Medicine;

J. Kappelmayer, MD, PhD, Department of Clinical Biochemistry and
Molecular Pathology; Z. Gyurcsik Némethné, Department of Physiotherapy, University of Debrecen MHSC; G. Szegedi, MD, PhD, Research Center for Autoimmune Diseases, Hungarian Academy of Sciences; Y. Shoenfeld, MD, PhD, Department of Medicine B and Center for Autoimmune Diseases, Sheba Medical Center; S. Sipka, MD, PhD, Laboratory of Immunology, Third Department of Medicine, Institute of Medicine; P. Soltész, MD, PhD, Cardiovascular Unit, Third Department of Medicine, Institute of Medicine; Z. Szekanecz, MD, PhD, Department of Rheumatology, Institute of Medicine; S. Szántó, MD, PhD, Department of Rheumatology, Institute of Medicine, University of Debrecen MHSC. Dr. Bodnár and Dr. Kerekes are co-first authors with equal contribution to this report.

Address correspondence to Prof. Z. Szekanecz, University of Debrecen, Medical and Health Science Center, Institute of Medicine, Department of Rheumatology, Nagyerdei str 98, H-4032 Debrecen, Hungary.

E-mail: szekanecz.zoltan@med.unideb.hu

Accepted for publication November 8, 2010.

Personal non-commercial use only. The Journal of Rheumatology Copyright @ (2011. All rights reserved. 
prevalence ratios of ischemic heart disease, atherosclerosis, and cerebrovascular disease were $1.2,1.5$, and 1.7, respectively, in AS in comparison to healthy controls ${ }^{11}$. There may be a 4.4-fold increased risk for myocardial infarction in $\mathrm{AS}^{8}$. The basic mechanisms of accelerated atherosclerosis and cardiovascular disease in AS are similar to those associated with $\mathrm{RA}^{1,4,5}$. Among traditional Framingham risk factors, AS has been associated with high prevalence of metabolic syndrome including dyslipidemia, high ratio of low-density lipoprotein cholesterol (LDL-C) to high-density lipoprotein cholesterol (HDL-C), and hyperhomocysteinemia ${ }^{12,24}$. However, inflammatory mechanisms underlying AS may be the key factors that lead to atherosclerosis and vascular disease. Sustained systemic inflammation in AS is accompanied by elevated serum levels of C-reactive protein (CRP), an acute-phase protein with proatherogenic effects ${ }^{13,17}$. Proinflammatory cytokines such as tumor necrosis factor- $\alpha$ (TNF- $\alpha$ ) may also be involved in this process, and TNF blockade leads to the improvement of lipid profile in $\mathrm{AS}^{19}$. TNF- $\alpha$ and CRP have been implicated in impaired coronary flow reserve and left ventricular diastolic function in $\mathrm{AS}^{10}$.

Noninvasive angiological methods have been developed to evaluate endothelial and vascular function in rheumatic as well as autoimmune diseases. Flow-mediated vasodilation (FMD), common carotid intima-media thickness (ccIMT), and pulse-wave velocity (PWV) are reliable indicators of endothelium-dependent vascular function, overt atherosclerosis, and arterial stiffness, respectively. We and others have used these techniques to assess abnormalities of vascular function in $\mathrm{RA}^{3,25,26,27}$. Similar methods can be applied to detect endothelial dysfunction, atherosclerosis, and vascular stiffness in $\mathrm{AS}^{6,7,8,10,13,15,17}$. There have been relatively few and somewhat controversial data regarding vascular function in $\mathrm{AS}^{23}$. There is a 1.5-times higher risk for atherosclerosis, a 1.2 to 4.4-times higher risk for ischemic heart disease, a 1.6-times higher risk for peripheral vascular disease, and a 1.7-times higher risk for cerebrovascular disease in AS. This increased risk is comparable to that reported in RA $(1.9,1.5,2.4 \text {, and 1.6-times risk, respectively })^{8,11}$. Chronic inflammation may be the major risk factor for atherosclerosis in $\mathrm{AS}^{14,23}$, as well as in other arthritides ${ }^{1,4,5,14}$.

There have been recent studies that separately assessed FMD, ccIMT, or PWV in AS. Endothelial dysfunction indicated by impaired FMD has been reported by Sari, et $a l^{17}$ in a cohort of 54 patients with AS. Impaired FMD did not correlate with age, sex, CRP, erythrocyte sedimentation rate (ESR), smoking habits, or disease activity. Pieringer ${ }^{15}$ also published preliminary results on impaired FMD in AS.

Sari, et al $^{17}$ also assessed ccIMT in the same cohort and found no differences between AS and the control group. Other groups, such as Malesci, et $a l^{12}$ and Choe, et $a l^{6}$, also reported normal ccIMT in cohorts of 24 to 28 patients with AS in comparison to healthy subjects. Gonzalez-Juanatey, et $a l^{7}$ assessed ccIMT and carotid plaques in 64 patients with
AS. Carotid plaques were more commonly observed in AS compared to controls. But no differences in ccIMT were observed. In contrast to these reports, Mathieu, et $a l^{13}$ reported a marginal, nonsignificant increase of ccIMT in 60 patients with AS compared to controls. Finally, Peters, et $a l^{8}$ assessed 59 patients with AS and found significantly higher ccIMT in the patients in comparison to controls even after adjustment for traditional cardiovascular risk factors.

Regarding arterial stiffness, Choe, et $a l^{6}$ estimated elastic properties including distensibility, stiffness index, and incremental elastic modulus, but not PWV, in 28 patients with AS. Arterial elastic measurements did not differ between patients with AS and controls. Moyssakis, et a ${ }^{28}$ evaluated aortic distensibility and reported impaired elasticity in AS. Mathieu, et $a l^{13}$ determined the augmentation index (AIx), another indicator of arterial stiffness, in 60 patients with AS and controls. There was a trend toward increased arterial stiffness in AS in comparison to controls; however, this difference was not statistically significant. To date, none of the investigators have reported data on PWV, a standard indicator of arterial stiffness in AS.

All these studies showed data from separate assessments of FMD, ccIMT, and elasticity. Only Sari, et al ${ }^{17}$ investigated FMD and ccIMT simultaneously, but they did not assess vascular stiffness. There have been no reports on PWV in AS. In addition, none of the investigators assessed the 3 vascular measurements (FMD, ccIMT, and PWV) together and also in association with numerous other clinical and laboratory measurements.

Therefore, we conducted a complex study and assessed FMD, ccIMT, and PWV in the same cohort of patients with AS. In addition, we correlated vascular function with several other clinical and laboratory measurements including age, disease duration, smoking, body mass index (BMI), disease activity, pain intensity determined by the patient [visual analog scale (VAS)], Bath AS Disease Activity Index (BASDAI), lumbar spine mobility, chest expansion, wall-occiput distance, Bath AS Functional Index (BASFI), CRP, ESR, and HLA-B27 status. Ours may be the first study that investigated endothelial function, atherosclerosis, and arterial stiffness in the same patient cohort and correlated these markers with numerous other indicators.

\section{MATERIALS AND METHODS}

Patients. The study patient group was 43 patients with AS [31 men (72\%), 12 women (28\%); mean age $45.4 \pm 11.8$ yrs, range $26-75$ years; all white; Table 1]. The diagnosis of AS was based on the modified New York criteria $^{29}$. Among the 43 patients, $33(76.7 \%)$ had only axial involvement, while $10(23.3 \%)$ also had peripheral arthritis. There were 36 patients $(83.7 \%)$ who were HLA-B27-positive. Fourteen of the 43 patients (32.6\%) were in an active state of disease $(\text { BASDAI }>40)^{30}$. Most patients $(37 / 43,86 \%)$ received nonsteroidal antiiflammatory drugs (NSAID). The possible cardiovascular effects of NSAID cannot be fully ruled out; however, as none of the patients with AS had clinical cardiovascular disease, this possible effect was not observed on the clinical level. Among the 10 patients with AS who had peripheral involvement, $6(60 \%)$ received conventional dis-

Personal non-commercial use only. The Journal of Rheumatology Copyright @ 2011 . All rights reserved. 
Table 1. Description of the ankylosing spondylitis population.

\begin{tabular}{lcc}
\hline Variable & Mean \pm SD & Range \\
\hline Age, yrs & $45.4 \pm 11.8$ & $26-75$ \\
Men:women ratio & $31: 12$ & - \\
Age at diagnosis, yrs & $32.8 \pm 10.5$ & $16-57$ \\
BMI, kg/m ${ }^{2}$ & $25.0 \pm 3.8$ & $19-33$ \\
Disease duration, yrs & $13.2 \pm 10.6$ & $2-40$ \\
Axial:peripheral ratio & $33: 10$ & - \\
ESR, mm/h & $15.5 \pm 15.6$ & $2-68$ \\
CRP, mg/l & $9.00 \pm 11.5$ & $0.5-56.7$ \\
HLA-B27 positivity, \% & 83.7 & - \\
Current smokers, \% & 30.2 & - \\
Pain on VAS, mm & $51.1 \pm 31.9$ & $12-90$ \\
Disease activity on VAS, mm & $49.7 \pm 28.5$ & $8-93$ \\
Active disease (\% BASDAI $>$ 40) & 32.6 & - \\
BASDAI, mm & $50.4 \pm 19.1$ & $19-80$ \\
BASFI & $45.4 \pm 11.8$ & \\
BASMI & $45.4 \pm 11.8$ & \\
Lumbar spine mobility; Schober, cm & $2.2 \pm 1.7$ & $0.5-5.5$ \\
Chest expansion, cm & $2.3 \pm 1.2$ & $1.1-4.5$ \\
Wall-occiput distance, cm & $8.2 \pm 8.5$ & $0-22$ \\
Current NSAID therapy, \% & 86 & - \\
Current DMARD therapy, \% & 14 & - \\
Current anti-TNF therapy, \% & 65 & - \\
\hline
\end{tabular}

BMI: body mass index; ESR: erythrocyte sedimentation rate; CRP: C-reactive protein; VAS: visual analog scale; BASDAI: Bath AS Disease Activity Index; BASFI: Bath AS Functional Index; BASMI: Bath AS Metrology Index; NSAID: nonsteroidal antiinflammatory drug; DMARD: disease-modifying antirheumatic drug; TNF: tumor necrosis factor.

ease-modifying antirheumatic drugs (DMARD) including methotrexate or sulfasalazine. Twenty-eight patients $(65.1 \%)$ took anti-TNF biologics. We recruited 40 age-matched and sex-matched healthy control subjects from volunteer hospital workers, visitors, and relatives [27 men (67.5\%) and 13 women (32.5\%); mean age $48.2 \pm 13.2 \mathrm{yrs}$, range $24-80 \mathrm{yrs}$; all white]. None of the patients with AS received systemic or local corticosteroids at the time of or at least 3 months prior to our study. None of the patients or controls received any vasoactive drugs or anticoagulants, such as aspirin, clopidogrel, heparin, warfarin/acenocoumarol, statins, angiotensin-converting enzyme inhibitors, or calcium channel blockers.

Each participant gave informed consent, according to the Declaration of Helsinki. For our study we also obtained local ethical committee approval at the University of Debrecen. Serum samples were then obtained from all subjects and kept frozen at $-70^{\circ} \mathrm{C}$ until further use.

Clinical and laboratory measurements. The age, disease duration, and BMI of all patients with AS were recorded. Pain intensity and disease activity were determined by the patient on a 10-cm VAS. Disease activity and functional capacity mobility were also tested by obtaining BASDAI and BASFI, respectively. Metric measurements including lumbar spine mobility were assessed by Schober's test; chest expansion and wall-occiput distance were also recorded.

Among laboratory indicators, ESR $(\mathrm{mm} / \mathrm{h})$ was assessed by the Westergren method. Serum CRP $(\mathrm{mg} / \mathrm{l})$ was measured by quantitative nephelometry (Roche Cobas Mira Plus, Block Scientific, Bohemia, NY, USA), using CRP reagents (Dialab, Vienna, Austria). After overnight fasting, blood samples were taken from the patients and controls for serum glucose, total cholesterol, LDL-C, HDL-C, triglyceride, renal and liver function tests, and full blood count. Urine samples were tested by Uricont-S. HLA-B27 genotyping was performed using polymerase chain reaction-sequence-specific primer technique (Histo Type B27 high resolution kit, BAG, Lich, Germany).
Assessment of possible cardiovascular involvement and study inclusion criteria. All patients with AS and healthy controls had a negative history for previous cardiovascular, cerebrovascular, or peripheral arterial disease. The AS and control groups were comparable in lipid levels, BMI, blood pressure, and other traditional cardiovascular risk factors (Table 2). Thirteen of the 43 patients with AS (30.2\%) and 14 of 40 controls (35.0\%) were current smokers. We applied the cutoff points published by Pedersen, $e t a l^{31}$ in RA ( $\geq 20$ pack-years). Cumulative tobacco intake was also calculated by multiplying the mean daily intake by the duration of consumption in years. Patients with traditional cardiovascular risk factors other than smoking, such as hypertension (blood pressure $>140 / 90 \mathrm{~mm} \mathrm{Hg}$ ), diabetes mellitus, and those with any vasculitis, current infectious disease or renal failure (serum creatinine $\geq 117 \mathrm{mmol} / \mathrm{l}$ ) were excluded. Cardiovascular involvement was also assessed by electrocardiogram (ECG) and echocardiography performed in all patients and controls. Based on all these data, all patients with AS or controls were considered free of clinically relevant vascular disease.

Assessment of brachial artery FMD. Brachial FMD and nitroglycerin-mediated vasodilation were assessed as described by us and oth$\mathrm{ers}^{25,32}$. Ultrasound examination was performed on the right arm using a 10 $\mathrm{MHz}$ linear array transducer (HP Sonos 5500 ultrasound system) by a single trained sonographer after $30 \mathrm{~min}$ resting in a temperature-controlled room (basal value for FMD). A B-mode longitudinal section was obtained of the brachial artery above the antecubital fossa. In order to assess FMD, reactive hyperemia was induced by release of a pneumatic cuff around the forearm inflated to suprasystolic pressure for $4.5 \mathrm{~min}$. After deflation, the maximal flow velocity and the arterial diameter was continuously recorded for $90 \mathrm{~min}$. Flow velocities, the baseline diameter, and FMD were ECGgated and detected offline. Three repeated FMD measurements were performed on each patient or control subject. Mean FMD values were expressed as percent change from baseline (resting) value.

Determination of common carotid atherosclerosis. The ccIMT measurements were carried out as described by us and others ${ }^{25,33}$. A duplex ultrasound system (HP Sonos 5500, $10 \mathrm{MHz}$ linear array transducer) was used to assess the common carotid arteries by a single observer. Longitudinal high-resolution B-mode ultrasound scans were employed over both right and left common carotid arteries and were R-synchronized and recorded. The offline measurements were performed $1 \mathrm{~cm}$ proximal to the carotid bulb in the far wall. Sites of carotid plaques were avoided. The ccIMT was defined as the distance between the first and second echogenic lines from the lumen, taking the average of 10 measurements on both sides. Values of ccIMT were expressed in $\mathrm{mm}$.

Assessment of $P W V$. Determination of arterial stiffness was carried out after a modification of our validated technique ${ }^{26}$. The assessment of PWV is

Table 2. Traditional cardiovascular risk factors in patients with ankylosing spondylitis (AS) and controls. Values are mean $\pm \mathrm{SD}$, except where indicated.

\begin{tabular}{lccc}
\hline Risk Factors & $\begin{array}{c}\text { AS, } \\
\mathrm{n}=43\end{array}$ & $\begin{array}{c}\text { Controls, } \\
\mathrm{n}=40\end{array}$ & $\mathrm{p}$ \\
\hline Age, yrs & $45.4 \pm 11.8$ & $48.2 \pm 13.2$ & 0.78 \\
Systolic blood pressure, $\mathrm{mm} \mathrm{Hg}$ & $130.7 \pm 11.8$ & $132.0 \pm 12.9$ & 0.82 \\
Diastolic blood pressure, $\mathrm{mm} \mathrm{Hg}$ & $86.1 \pm 9.2$ & $84.3 \pm 6.9$ & 0.75 \\
Total cholesterol, mmol/1 & $5.52 \pm 1.22$ & $5.45 \pm 0.91$ & 0.69 \\
LDL cholesterol, mmol/l & $3.25 \pm 1.03$ & $3.30 \pm 0.82$ & 0.91 \\
HDL cholesterol, mmol/l & $1.58 \pm 0.55$ & $1.65 \pm 0.39$ & 0.77 \\
Triglyceride, mmol/1 & $1.49 \pm 0.69$ & $1.39 \pm 0.78$ & 0.68 \\
BMI, kg/m 2 & $25.0 \pm 3.8$ & $24.7 \pm 4.8$ & 0.88 \\
Current smokers, \% & 30.2 & 35.0 & - \\
\hline
\end{tabular}

LDL: low-density lipoprotein; HDL: high-density lipoprotein; BMI: body mass index.

Personal non-commercial use only. The Journal of Rheumatology Copyright @ 2011 . All rights reserved. 
based on the contraction of the myocardium initiating pulse waves in the aorta. The first wave becomes reflected from the aortic wall at the bifurcation; therefore a second, reflected wave appears as a late systolic peak. The morphology of this second, reflected wave depends on the stiffness of the large artery. Thus, we acquired suprasternal and femoral images and pulse-wave Doppler signals (HP Sonos 5500, 2-4 MHz phased array and 5-10 MHz linear array transducers). Simultaneous ECG recording was performed. ECG-gated Doppler analyses were performed over the beginning of the descending aorta and over the common femoral artery at the level of the inguinal ligament. Pulse-wave Doppler signals were recorded over 10 cardiac cycles at $150 \mathrm{~mm} / \mathrm{s}$ sweep speed. Distances between the suprasternal notch and the 2 sampling sites were also measured and pulse transit times were recorded. The time delay was derived from the difference between the 2 transit times and PWV in $\mathrm{m} / \mathrm{s}$ was calculated as distance divided by time delay.

In order to have reproducible results, all subjects needed a rest for at least 5 min prior to FMD and PWV assessments.

Statistical analysis and reproducibility. For the analysis of FMD and PWV, Kolmogorov-Smirnov and Lilliefors tests were used. Subsequently we performed correlation analyses. In cases of normal distribution (parametric), Pearson's test was performed, while in cases of non-normal distribution (nonparametric), Spearman's test was performed. R values of these correlations were determined and corresponding $\mathrm{p}$ values $<0.05$ were considered significant.

Regarding reproducibility, all assessments were performed by a single observer. Intraobserver variability of FMD, ccIMT, and PWV measurements were calculated as $5 \%, 4.2 \%$, and $3.3 \%$, respectively. The "stability" of measurements is indicated by the reproducibility for month-to-month repeated assessments of FMD, ccIMT, or PWV. According to the Brand-Altman analysis, the $95 \%$ limits of agreement ranged between $-1.6 \%$ and $1.9 \%$ for all assessments.

\section{RESULTS}

Assessment of FMD, ccIMT, and PWV. In order to assess endothelial function, brachial artery FMD was measured by high-resolution ultrasonography. FMD in patients with AS expressed in percentage of the basal value was significantly lower $(6.85 \pm 2.98 \%)$ in comparison to controls $(8.30 \pm$ $3.96 \% ; \mathrm{p}=0.005$; Figure 1A).

A duplex ultrasound system was used to assess ccIMT. In patients with AS $(0.65 \pm 0.15 \mathrm{~mm})$, ccIMT was significantly higher in comparison to controls $(0.54 \pm 0.15 \mathrm{~mm}$; $\mathrm{p}=$ 0.01 ; Figure 1B).

$\mathrm{PWV}$, an indicator of aortic stiffness, was significantly increased in patients with AS $(8.64 \pm 2.44 \mathrm{~m} / \mathrm{s})$ in comparison to controls $(8.00 \pm 1.46 \mathrm{~m} / \mathrm{s} ; \mathrm{p}=0.03$; Figure $1 \mathrm{C})$.

Correlations between FMD, ccIMT, PWV, and clinical and laboratory markers in patients with AS. In patients with AS, values of FMD, ccIMT, and PWV were correlated with each other, as well as with other clinical and laboratory indicators (Table 2).

FMD and ccIMT negatively correlated with each other $(\mathrm{r}=-0.563 ; \mathrm{p}=0.0001)$. PWV also correlated with ccIMT $(\mathrm{r}=0.374 ; \mathrm{p}=0.018$; Table 3$)$.

Both ccIMT and PWV exerted positive correlations with disease duration $(\mathrm{r}=0.559 ; \mathrm{p}=0.013$ and $\mathrm{r}=0.520 ; \mathrm{p}=$ 0.022 , respectively), but FMD did not. PWV also correlated with age $(r=0.382 ; \mathrm{p}=0.016)$. Higher ccIMT or PWV also correlated with increased BASFI $(r=0.691 ; p=0.003$ and
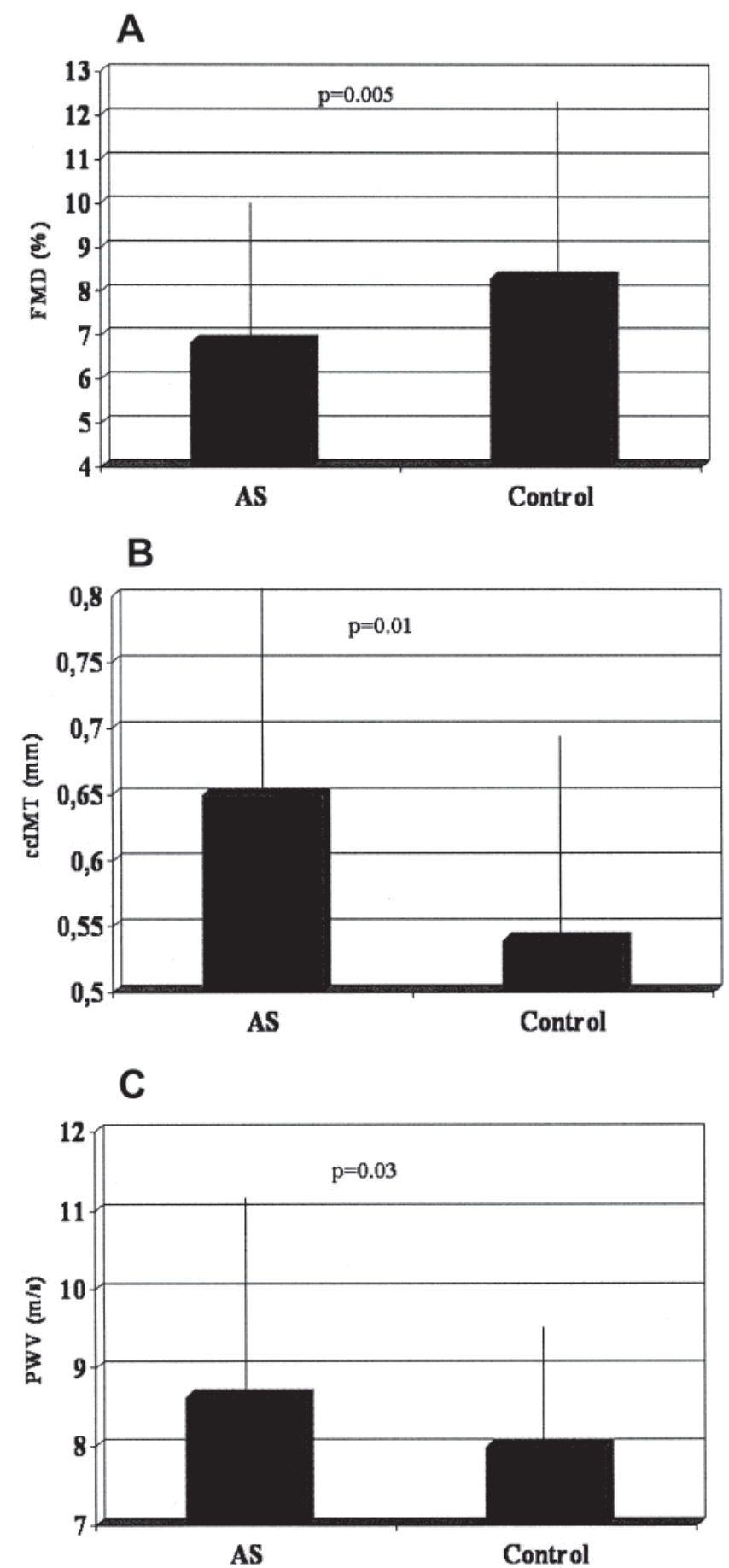

Figure 1. Measures of endothelial dysfunction, carotid atherosclerosis, and aortic stiffness in patients with ankylosing spondylitis (AS; $n=43)$ and healthy controls $(n=40)$. A. Mean flow-mediated vasodilation (FMD; percentage). B. Common carotid intima-media thickness (ccIMT; mm). C. Pulse-wave velocity (PWV; $\mathrm{m} / \mathrm{s}$ ). There is significant impairment of FMD as well as significantly increased ccIMT and PWV in patients with AS in comparison to controls.

$\mathrm{r}=0.654 ; \mathrm{p}=0.006)$, negatively correlated with lumbar spine mobility $(\mathrm{r}=-0.656 ; \mathrm{p}=0.006$ and $\mathrm{r}=-0.604 ; \mathrm{p}=$ $0.013)$ and chest expansion $(\mathrm{r}=-0.502 ; \mathrm{p}=0.047$ and $\mathrm{r}=$ 
Table 3. Relevant correlations between vascular and other measurements in patients with ankylosing spondylitis $(n=43)$.

\begin{tabular}{lccc}
\hline Vascular Measurements & $\begin{array}{c}\text { Clinical/Laboratory } \\
\text { Measurements }\end{array}$ & $\mathrm{r}$ & $\mathrm{p}$ \\
\hline ccIMT & FMD & -0.563 & 0.0001 \\
ccIMT & PWV & 0.374 & 0.018 \\
ccIMT & Disease duration & 0.559 & 0.013 \\
ccIMT & BASFI & 0.691 & 0.003 \\
ccIMT & Lumbar spine mobility & -0.656 & 0.006 \\
ccIMT & Chest expansion & -0.502 & 0.047 \\
ccIMT & Wall-occiput distance & 0.509 & 0.044 \\
PWV & Age & 0.382 & 0.016 \\
PWV & Disease duration & 0.520 & 0.022 \\
PWV & Lumbar spine mobility & -0.604 & 0.013 \\
PWV & Chest expansion & -0.613 & 0.012 \\
PWV & Wall-occiput distance & 0.614 & 0.011
\end{tabular}

ccIMT: common carotid intima-media thickness; FMD: flow-mediated vasodilation; PWV: pulse-wave velocity; BASFI: Bath AS Functional Index.

$-0.613 ; \mathrm{p}=0.012)$ and positively correlated with wall-occiput distance $(\mathrm{r}=0.509 ; \mathrm{p}=0.044$ and $\mathrm{r}=0.614$; $\mathrm{p}=0.011$; Table 3 ). FMD did not correlate with any functional or metric measurements (data not shown).

As an internal control, the widely used disease activity scale BASDAI strongly correlated with the patient's assessment of activity on VAS $(r=0.922 ; p=0.0001)$, indicating that patient's VAS may be a simple and useful assessment tool in this respect. However, none of the vascular measurements showed any correlation with disease activity markers, such as BASDAI, ESR, CRP, or patient's assessment of activity on VAS (data not shown). Further, no significant associations were observed in patients with AS among FMD, ccIMT, or PWV values in comparison to sex distribution, drug treatment modalities, BMI, HLA-B27 status, current smoking, or patient's assessment of pain on VAS (data not shown).

\section{DISCUSSION}

Cardiovascular and cerebrovascular diseases are major causes of morbidity and mortality in the general population, as well as in autoimmune-inflammatory diseases ${ }^{1,2,4,5}$. Early identification of patients with higher risk for vascular disorders allows us to introduce primary prevention or effective pharmacological treatment. Assessing risk for future vascular events involves measuring FMD, overt ccIMT, and $\mathrm{PWV}^{25,26,32,33,34}$. There have been numerous recent studies showing increased ccIMT and PWV as well as impaired

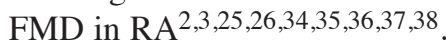

There have been relatively few and somewhat controversial data regarding vascular function in $\mathrm{AS}^{23}$. Most recent studies separately assessed FMD, ccIMT, or PWV in AS. Sari, et $a l^{17}$ and Pieringer ${ }^{15}$ found impaired FMD in AS. We also found impaired FMD in 43 patients with AS in comparison to controls. In addition, decreased FMD correlated with increased ccIMT in AS. We found no associations between FMD and functional or metric measurements of AS, suggesting that FMD is a "snapshot" of endothelial function, while structural and functional damage of the musculoskeletal system occurs after many years.

Most groups, including Sari, et $a l^{17}$, Malesci, et $a l^{12}$, and Choe, et $a l^{6}$, did not find any differences in ccIMT between AS and control groups. These AS cohorts consisted of 26-28 patients. Gonzalez-Juanatey, et $a l^{7}$ reported more carotid plaques, but unchanged ccIMT in AS. In contrast, similar to our results, Mathieu, et $a l^{13}$ and Peters, et $a l^{8}$ found significantly higher ccIMT in AS compared to controls. In the latter study, more than 50 patients with AS were recruited. We also report significantly increased ccIMT in AS compared to controls. Our results are similar to those published by Peters, et $a l^{8}$, but are different from the other studies. The studies of Malesci, et $a l^{12}$ and Choe, et $a l^{6}$ included far fewer patients than our study. Although Sari, et $a l^{17}$ reported normal ccIMT in patients with AS, there could be other differences between that cohort and ours. Also, Sari, et $a l^{17}$ published their data in 2006, while Peters, et al ${ }^{8}$ and Mathieu, et $a l^{13}$ reported results that are very similar to ours in 2008-2009. Further studies are needed to come to a decision regarding carotid atherosclerosis in AS. Regarding possible correlations, ccIMT correlated with impaired FMD and increased aortic stiffness and also correlated with disease duration, BASFI, and metric measurements including lumbar spine mobility, chest expansion, and wall-occiput distance. Other investigators assessing ccIMT did not report associations of ccIMT with any of these functional or metric measurements. Our results indicate that the development of atherosclerosis occurs over a longer period of time and is associated with longer duration of AS and the development of more severe structural and functional disability.

There have been absolutely no data on PWV. Choe, et al reported normal elasticity while Moyssakis, et $a l^{28}$ found impaired elasticity in AS. Mathieu, et $a l^{13}$ found nonsignificant increase of AIx in AS compared to controls. Our study is the first showing PWV data in AS. PWV may be the most widely determined and most reliable indicator of arterial stiffness $^{26,39}$. We found significantly increased aortic stiffness indicated by increased PWV in patients with AS compared to healthy controls. Moreover, PWV correlated with ccIMT, as well as with age, disease duration, BASFI, and all metric measurements described. Thus, aortic stiffness, similarly to carotid atherosclerosis, develops in parallel with the progression of AS. Further, because PWV was correlated with ccIMT, increased stiffness may be a consequence of atherosclerosis.

There have been reports on endothelial dysfunction, carotid atherosclerosis, and arterial stiffness in AS. However, all investigator groups assessed FMD, ccIMT, or stiffness separately. Only Sari, et $a l^{17}$ evaluated FMD and ccIMT in the same cohort, but arterial stiffness was not recorded. Our results on impaired FMD in AS correspond

Personal non-commercial use only. The Journal of Rheumatology Copyright @ $\subset$ 2011. All rights reserved. 
with those published in the only available larger AS study, performed by Sari, et al ${ }^{17}$. There have been controversies regarding ccIMT studies. While normal ccIMT was found in some studies, one of the most recent reports by Peters, et $\mathrm{ll}^{8}$, as well as our study, described increased ccIMT in AS. This controversial issue needs to be studied further. Regarding stiffness, we are the first group that assessed PWV rather than elasticity or AIx in AS. PWV has become the most reliable marker of vascular stiffness in recent studies ${ }^{26,35,40,41}$. Therefore, ours is the first study that assessed FMD, ccIMT, and PWV simultaneously, in the same AS cohort. In addition, we performed extensive correlation analysis in the patients with AS. Endothelial dysfunction indicated by impaired FMD may be a transient, short-term event that may precede overt atherosclerosis and vascular disease. Thus, FMD could not be correlated with disease duration and chronic disability resulting from AS. In contrast, we are the first to report significant correlations between carotid atherosclerosis and aortic stiffness with AS disease duration and functional and metric impairment. None of the assessed vascular measurements correlated with any markers of disease activity in AS.

The strength of our study is that we include 3 noninvasive techniques to detect endothelial function, atherosclerosis, and stiffness in the same AS population. Further, we correlated these markers with numerous clinical and laboratory measurements. A limitation of our study could be the relatively low patient number, although even smaller cohorts were studied by others. We also cannot fully exclude the possible confounding effects of medications, such as NSAID or anti-TNF biologics.

Thus, patients with AS may be screened for atherosclerosis and subclinical vasculopathy using these noninvasive techniques. Patients at high risk should undergo primary or secondary prevention. Statins might be beneficial for patients with $\mathrm{AS}^{18}$. There have been many reports on the possible vasculoprotective effects of anti-TNF biologics in $\mathrm{RA}^{42,43}$. Reports have suggested that TNF blockade might improve dyslipidemia and microvascular function in $\mathrm{AS}^{19,20}$. Ten evidence-based recommendations for the cardiovascular management of RA, AS, and patients with psoriatic arthritis have been developed by a European League Against Rheumatism task force ${ }^{3}$. It is recommended that every effort should be made to identify high-risk patients early. Moreover, the aggressive suppression of systemic inflammation by DMARD or biologics may also lower the risk for vascular diseases in $\mathrm{AS}^{3}$.

\section{REFERENCES}

1. Szekanecz Z, Kerekes G, Der H, Sandor Z, Szabo Z, Vegvari A, et al. Accelerated atherosclerosis in rheumatoid arthritis. Ann NY Acad Sci 2007;1108:349-58.

2. Gonzalez-Gay MA, Gonzalez-Juanatey C, Martin J. Rheumatoid arthritis: a disease associated with accelerated atherogenesis. Semin Arthritis Rheum 2005;35:8-17.
3. Peters MJ, Symmons DP, McCarey D, Dijkmans BA, Nicola P, Kvien TK, et al. EULAR evidence-based recommendations for cardiovascular risk management in patients with rheumatoid arthritis and other forms of inflammatory arthritis. Ann Rheum Dis 2010;69:325-31

4. Szekanecz Z, Koch AE. Vascular involvement in rheumatic diseases: 'vascular rheumatology'. Arthritis Res Ther 2008;10:224.

5. Sherer Y, Shoenfeld Y. Mechanisms of disease: atherosclerosis in autoimmune diseases. Nat Clin Pract Rheumatol 2006;2:99-106.

6. Choe JY, Lee MY, Rheem I, Rhee MY, Park SH, Kim SK. No differences of carotid intima-media thickness between young patients with ankylosing spondylitis and healthy controls. Joint Bone Spine 2008;75:548-53.

7. Gonzalez-Juanatey C, Vazquez-Rodriguez TR, Miranda-Filloy JA, Dierssen T, Vaqueiro I, Blanco R, et al. The high prevalence of subclinical atherosclerosis in patients with ankylosing spondylitis without clinically evident cardiovascular disease. Medicine 2009;88:358-65.

8. Peters MJ, van Eijk IC, Smulders YM, Serne E, Dijkmans BA, van der Horst-Bruinsma IE, et al. Signs of accelerated preclinical atherosclerosis in patients with ankylosing spondylitis. J Rheumatol 2010;37:161-6.

9. Peters MJ, Visman I, Nielen MM, Van Dillen N, Verheij RA, van der Horst-Bruinsma IE, et al. Ankylosing spondylitis: a risk factor for myocardial infarction? Ann Rheum Dis 2010;69:579-81.

10. Caliskan M, Erdogan D, Gullu H, Yilmaz S, Gursoy Y, Yildirir A, et al. Impaired coronary microvascular and left ventricular diastolic functions in patients with ankylosing spondylitis. Atherosclerosis 2008;196:306-12.

11. Han C, Robinson DW Jr, Hackett MV, Paramore LC, Fraeman KH, Bala MV. Cardiovascular disease and risk factors in patients with rheumatoid arthritis, psoriatic arthritis, and ankylosing spondylitis. J Rheumatol 2006;33:2167-72.

12. Malesci D, Niglio A, Mennillo GA, Buono R, Valentini G, La Montagna G. High prevalence of metabolic syndrome in patients with ankylosing spondylitis. Clin Rheumatol 2007;26:710-4.

13. Mathieu S, Joly H, Baron G, Tournadre A, Dubost JJ, Ristori JM, et al. Trend towards increased arterial stiffness or intima-media thickness in ankylosing spondylitis patients without clinically evident cardiovascular disease. Rheumatology 2008;47:1203-7.

14. Peters MJ, van der Horst-Bruinsma IE, Dijkmans BA, Nurmohamed MT. Cardiovascular risk profile of patients with spondylarthropathies, particularly ankylosing spondylitis and psoriatic arthritis. Semin Arthritis Rheum 2004;34:585-92.

15. Pieringer $\mathrm{H}$. Impaired endothelial function in patients with ankylosing spondylitis. Rheumatology 2006;45:1319.

16. Sari I, Kebapcilar L, Alacacioglu A, Bilgir O, Yildiz Y, Taylan A, et al. Increased levels of asymmetric dimethylarginine (ADMA) in patients with ankylosing spondylitis. Intern Med 2009;48:1363-8.

17. Sari I, Okan T, Akar S, Cece H, Altay C, Secil M, et al. Impaired endothelial function in patients with ankylosing spondylitis. Rheumatology 2006;45:283-6.

18. van Denderen JC, Peters MJ, van Halm VP, van der Horst-Bruinsma IE, Dijkmans BA, Nurmohamed MT. Statin therapy might be beneficial for patients with ankylosing spondylitis. Ann Rheum Dis 2006;65:695-6.

19. van Eijk IC, de Vries MK, Levels JH, Peters MJ, Huizer EE, Dijkmans BA, et al. Improvement of lipid profile is accompanied by atheroprotective alterations in high-density lipoprotein composition upon tumor necrosis factor blockade: a prospective cohort study in ankylosing spondylitis. Arthritis Rheum 2009;60:1324-30.

20. van Eijk IC, Peters MJ, Serne EH, van der Horst-Bruinsma IE, Dijkmans BA, Smulders YM, et al. Microvascular function is impaired in ankylosing spondylitis and improves after tumour 
necrosis factor alpha blockade. Ann Rheum Dis 2009;68:362-6.

21. van Halm VP, van Denderen JC, Peters MJ, Twisk JW, van der Paardt M, van der Horst-Bruinsma IE, et al. Increased disease activity is associated with a deteriorated lipid profile in patients with ankylosing spondylitis. Ann Rheum Dis 2006;65:1473-7.

22. Zochling J, Braun J. Mortality in ankylosing spondylitis. Clin Exp Rheumatol 2008;5 Suppl 51:S80-4.

23. Heeneman S, Daemen MJ. Cardiovascular risks in spondyloarthritides. Curr Opin Rheumatol 2007;19:358-62.

24. Baskan BM, Sivas F, Aktekin LA, Dogan YP, Ozoran K, Bodur H. Serum homocysteine level in patients with ankylosing spondylitis. Rheumatol Int 2009;29:1435-9.

25. Kerekes G, Szekanecz Z, Der H, Sandor Z, Lakos G, Muszbek L, et al. Endothelial dysfunction and atherosclerosis in rheumatoid arthritis: a multiparametric analysis using imaging techniques and laboratory markers of inflammation and autoimmunity. J Rheumatol 2008;35:398-406.

26. Soltesz P, Der H, Kerekes G, Szodoray P, Szücs G, Danko K, et al. A comparative study of arterial stiffness, flow-mediated vasodilation of the brachial artery, and the thickness of the carotid artery intima-media in patients with systemic autoimmune diseases. Clin Rheumatol 2009;28:655-62.

27. Gonzalez-Gay MA, Gonzalez-Juanatey C, Ollier WE. Endothelial dysfunction in rheumatoid arthritis: influence of HLA-DRB1 alleles. Autoimmun Rev 2004;3:301-4

28. Moyssakis I, Gialafos E, Vassiliou VA, Boki K, Votteas V, Sfikakis PP, et al. Myocardial performance and aortic elasticity are impaired in patients with ankylosing spondylitis. Scand J Rheumatol 2009;38:216-21.

29. van der Linden S, Valkenburg HA, Cats A. Evaluation of diagnostic criteria for ankylosing spondylitis. A proposal for modification of the New York criteria. Arthritis Rheum 1984;27:361-8.

30. Garrett S, Jenkinson T, Kennedy LG, Whitelock H, Gaisford P, Calin A. A new approach to defining disease status in ankylosing spondylitis: the Bath Ankylosing Spondylitis Disease Activity Index. J Rheumatol 1994;21:2286-91.

31. Pedersen M, Jacobsen S, Garred P, Madsen HO, Klarlund M, Svejgaard A, et al. Strong combined gene-environment effects in anti-cyclic citrullinated peptide-positive rheumatoid arthritis: a nationwide case-control study in Denmark. Arthritis Rheum 2007;56:1446-53.

32. Corretti MC, Anderson TJ, Benjamin EJ, Celermajer D, Charbonneau F, Creager MA, et al. Guidelines for the ultrasound assessment of endothelial-dependent flow-mediated vasodilation of the brachial artery: a report of the International Brachial Artery Reactivity Task Force. J Am Coll Cardiol 2002;39:257-65.
33. Kanters SD, Algra A, van Leeuwen MS, Banga JD. Reproducibility of in vivo carotid intima-media thickness measurements: a review. Stroke 1997;28:665-71.

34. Gonzalez-Gay MA, Gonzalez-Juanatey C, Vazquez-Rodriguez TR, Martin J, Llorca J. Endothelial dysfunction, carotid intima-media thickness, and accelerated atherosclerosis in rheumatoid arthritis. Semin Arthritis Rheum 2008;38:67-70.

35. Maki-Petaja KM, Hall FC, Booth AD, Wallace SM, Yasmin H, Bearcroft PW, et al. Rheumatoid arthritis is associated with increased aortic pulse-wave velocity, which is reduced by anti-tumor necrosis factor-alpha therapy. Circulation 2006;114:1185-92.

36. Gonzalez-Juanatey C, Llorca J, Vazquez-Rodriguez TR, Diaz-Varela N, Garcia-Quiroga H, Gonzalez-Gay MA. Short-term improvement of endothelial function in rituximab-treated rheumatoid arthritis patients refractory to tumor necrosis factor alpha blocker therapy. Arthritis Rheum 2008;59:1821-4.

37. del Rincon I, Freeman GL, Haas RW, O'Leary DH, Escalante A. Relative contribution of cardiovascular risk factors and rheumatoid arthritis clinical manifestations to atherosclerosis. Arthritis Rheum 2005;52:3413-23

38. Salmon JE, Roman MJ. Subclinical atherosclerosis in rheumatoid arthritis and systemic lupus erythematosus. Am J Med 2008;10 Suppl 1:S3-8

39. Baulmann J, Schillings U, Rickert S, Uen S, Dusing R, Illyes M, et al. A new oscillometric method for assessment of arterial stiffness: comparison with tonometric and piezo-electronic methods. J Hypertens 2008;26:523-8.

40. Argyropoulou MI, Kiortsis DN, Daskas N, Xydis V, Mavridis A, Efremidis SC, et al. Distensibility and pulse wave velocity of the thoracic aorta in patients with juvenile idiopathic arthritis: an MRI study. Clin Exp Rheumatol 2003;21:794-7.

41. Inaba M, Tanaka K, Goto H, Sakai S, Yamada S, Naka H, et al. Independent association of increased trunk fat with increased arterial stiffening in postmenopausal patients with rheumatoid arthritis. J Rheumatol 2007;34:290-5.

42. Szekanecz Z, Kerekes G, Soltesz P. Vascular effects of biologic agents in RA and spondyloarthropathies. Nat Rev Rheumatol 2009;5:677-84.

43. Kerekes G, Soltesz P, Der H, Veres K, Szabo Z, Vegvari A, et al. Effects of biologics on vascular function and atherosclerosis associated with rheumatoid arthritis. Ann NY Acad Sci 2009;1173:814-21. 